\title{
AGE, MENOPAUSAL STATUS AND THE BLADDER MICROBIOME
}

Natasha Curtiss ${ }^{1}$, Aswini Balachandran ${ }^{1}$, Louise Krska², Claire Peppiatt-Wildman², Scott Wildman ${ }^{2}$, Jonathan Duckett ${ }^{1}$

${ }^{1}$ Department of Obstetrics and Gynaecology, Medway Maritime Hospital, Gillingham, Kent, UK. ME7 5NY

2 Urinary-System physiology Unit, Medway school of Pharmacy, Universities of Kent ME4 4TB

Corresponding Author:

Prof Jonathan Duckett MB ChB MD(Res) FRCOG, Consultant Urogynaecologist

Medway Maritime Hospital, Windmill Road, Gillingham, Kent, UK. ME7 5NY

Tele no: 01634835154 Fax no: 01634835155

Email address: jraduckett@hotmail.com

Conflicts of interest - none

$\mathrm{N}$ Curtiss collected and processed samples and wrote the manuscript

A Balachandran collected the samples and edited the manuscript

L Krska processed the samples and edited the manuscript

S Wildman developed the concept and edited the manuscript

J Duckett developed the concept and edited the manuscript 


\section{AGE, MENOPAUSAL STATUS AND THE BLADDER MICROBIOME}

Natasha Curtiss, Aswini Balachandran, Louise Krska, Claire Peppiatt-Wildman, Scott Wildman, Jonathan Duckett

\section{Abstract}

Objectives: The bladder is not sterile but contains a healthy community of microbes termed the microbiome. Alterations in the bladder microbiome have been demonstrated in disease states such as the overactive bladder. The microbiome in other anatomical niches is known to alter with age eg the vagina. The objective of this study was to identify if the bladder microbiome in healthy women varies with age and menopausal status.

Study design: Urine from 79 healthy women attending secondary care gynaecological clinics with no urinary symptoms provided clean catch mid-stream urine specimens. Urine was centrifuged and the resultant pellet was re-suspended and inoculated onto chocolate agar plates and cultured under either aerobic or anaerobic conditions. Morphologically different colonies were purity plated and 16s rRNA gene sequencing was performed. A microbe genomic basic local alignment search tool (BLAST) was used to identify the genus of the bacteria.

Results: There was no significant correlation between the age of a woman and the number of different genera identified $(r=-0.034, p=0.79)$. There were few significant differences in the frequency with which the majority of organisms were found in pre and post-menopausal women. The exceptions however were lactobacillus, which was more common in premenopausal women ( 31 vs $3 \mathrm{p}=0.002$ ) and Mobiluncus, which was more common in postmenopausal women ( 0 vs $3 p=0.02)$. 
Conclusions: There was no significant correlation between patient age and diversity of the bladder microbiome but large numbers of different organisms were identified. Significant differences were however observed for Lactobacillus which is more common in premenopausal women and Mobiluncus which is more common in in post-menopausal women.

Words 262

Keywords:

Lactobacillus, Microbiome, Overactive Bladder, Women 


\section{Introduction}

Several different centres around the world have established the presence of a bladder microbiome $[1,2]$. This complex interaction between microbes and host has been shown to be different in healthy individuals compared to those with a disease process such as $O A B$ $[3,4]$. The microbiome is known to alter in other body systems with external factors. The gut microbiome changes with external factors and also with age [5]. The vaginal microbiome also alters with age and estrogenisation [6]. The vagina is anatomically closely related to the bladder and any change in the vaginal microbiome might be expected to be reflected in changes in the bladder microbiome. It stands to reason that the bladder microbiome likewise will alter with age and menopausal status. The only previous study on the bladder microbiome and age include only 6 men and 10 women. This small study found the microbiome appears to alter with age [2] around a core microbiome but was too small to draw statistical conclusions on the changes of the bladder microbiome with age. This study was designed to test the hypothesis that the bladder microbiome changes with age and menopausal status. 


\section{Materials and Methods}

The microbiome of 79 healthy women attending a secondary care general gynaecology clinic and members of staff were included. This was a consecutive cohort study with patients compared by age. Ethical approval was registered (ClinicalTrials.gov: NCT 02536872). This study used previously collected control data from earlier work on the bladder microbiome in overactive bladder (35 cases) [7] with additional cases (44) to increase power. Inclusion criteria included patients/staff who did not score on the ICIQ short form questionnaire (International consultation on incontinence modular questionnaire - Urinary incontinence short form). Women using hormonal medication such as the progesterone only contraceptives (9 patients) or hormone replacement (2) were excluded. Patients taking medication to down regulate hormone production were also excluded (2). There were no combined contraceptive pill users in the cohort. Volunteers provided a clean catch voided urine sample into a sterile container. The samples were immediately tested for nitrites (Siemens Multistix@8SG), and any patients testing positive were excluded.

A pellet was formed from $5 \mathrm{ml}$ of each urine sample by centrifuging it at $800 \mathrm{rpm}$ for 5 mins. This pellet was re-suspended in $50 \mu \mathrm{l}$ of the supernatant and $5 \mu \mathrm{l}$ inoculated onto two chocolate agar plates ( $\mathrm{E}$ and $\mathrm{O}$ laboratories) by streaking. The plates were incubated at $37^{\circ} \mathrm{C}$, one in $5 \% \mathrm{CO} 2$ (48 hours) and the other in anaerobic conditions ( 7 days). Following incubation, morphologically different colonies were taken and purity plated on chocolate agar plates for 48 hours using the same conditions for growth. Aseptic techniques were employed throughout. Sequencing of the 16s rRNA gene was performed as follows. An Eppendorf Mastercycler set to $94^{\circ} \mathrm{C}$ for 5 minutes was used to lysis bacteria from $5 \mu \mathrm{l}$ inoculations of isolates suspended in $100 \mu \mathrm{l}$ sterile nuclease free water (Thermo Scientific). 
Lysed bacterial samples underwent polymerase chain reaction (PCR) to amplify a variable region of the bacterial 16rRNA gene. The primers used were; (DG74Fwd - 5'-AGG AGC TGA TCC AAC CGC A-3' - Eurofins) and (RDR080Rev - 5'-AAC TGG AGG AAG GTG GGG AC-3'Eurofins). A 1\% agarose gel loaded with the PCR amplicons was checked for reaction success against a negative control.

A PCR Purification Kit (Qiagen) was used to purifiy the DNA. The concentration of each product was measured using a Nanodrop 2000 (Thermo Scientific). Sequencing was performed by Eurofins Genomics, Ebersburg, Germany. A microbe genomic BLAST (Basic Local Alignment Search Tool, PubMed) was used to identify the genus of bacteria from the sequences. Some sequences could not be identified and were excluded from further analysis.

16s rRNA gene sequence information was used to compare the microbiomes across all patients. Patients were catagorised as pre-menopausal and post-menopausal and differences in their microbiomes were analysed by Fisher's Exact test. Microbiomes were also analysed by age using Pearson Correlation coefficient. A formal power calculation was not performed as this was a proof of concept study and sought to evaluate associations reported in previous studies. Sample size calculations are difficult when means and standard deviations are uncertain. A minimum sample size of more than 25 in each arm has been recommended [8]. 


\section{Results}

The urine from 79 women was analysed. In total 60 different genera were identified in the urine from the women analysed.

The diversity of the bladder microbiome was investigated by plotting age against number of genera identified (fig 1). There was not a significant correlation between the age of a woman and the number of different genera identified (statistical analysis using Pearson Correlation Coefficient $r=-0.034 p=0.79$ ). There is a trend towards decreased numbers of different genera in urine from post-menopausal women but this did not reach statistical significance.

There were few significant differences in the frequency at which majority of organisms were found in the microbiome of pre-menopaual and post-menopausal women. The exceptions were lactobacillus which was significantly more common in pre-menopausal women than post-menopausal women (31 pre-menopausal vs 3 post-menopausal $p=0.002$ ) and Mobiluncus which was more common in postmenopausal women (3 post-menopausal vs 0 pre-menopausal $p=0.02$ ). The distribution of the genera identified in pre and postmenopausal women are depicted in figure 2 . We identified a decrease in the growth of lactobacillus from urine as the age of women increased see table 1. 


\section{Comment}

In common with smaller studies we found no correlation between age and diversity as measured by number of genera identified [2]. There was a significantly greater incidence of Lactobacillus in the bladder microbiome of pre-menopausal women than post-menopausal women $(p=0.003)$ and Mobiluncus was significantly more common in postmenopausal women $(p=0.02)$. There were differences in the genera identified in the bladder of premenopausal women and post-menopausal women with some genera common to both.

This study adds valuable information to the developing understanding of the female urinary microbiome. Our results along with other studies are developing a picture of the core female urinary microbiome (FUM) [9]. By establishing the norm, we may increasingly be able to detect differences in bacterial colonisation of the bladder in health and disease with a view to manipulating the microbiome therapeutically to restore homeostasis. We identified 60 different genera across all patients. Some were found exclusively in younger and older women but a core of genera were found in all age groups. The bacteria most commonly found in urine by many research groups; Streptococcus, staphylococcus, Corynebacterium and lactobacillus $[1,3,10]$ are all within this core (fig 2 ).

Finding older women with no urinary symptoms was difficult and we were only able to recruit 19 suitable women in our study. Difficulties in obtaining age matched controls is not a new phenomenon and has been described by previous researchers [11]. It is well documented that there is an increase in the prevalence of OAB with age, and women attending the general gynaecology clinic after the menopause with no symptoms of prolapse or urinary symptoms is a rare occurrence. In light of this, general conclusions are 
more difficult to make and some subtle changes in the bladder microbiome may remain undetected.

There is a trend towards decreased numbers of different genera in-post menopausal specimens that did not reach statistical significance in this study, perhaps due to sample number. This concept of decreased numbers of genera is not unique to the urinary tract system, studies looking at the effect of ageing on the gut microbiome shows an overall loss in diversity alongside changes in the make up of the microbiome with increasing numbers of facultative anaerobes [12]. Data from another study where next generation sequencing was employed to investigate the bladder microbiome albeit in a small number of patinets mirrored our results. On average less genera were identified in women over 50 years old and a decrease in overall bacterial load was noted in this group compared to women aged 20-49 [2]. Unfortunately, our method is not quantitative and no comment can be made regarding how the overall bacterial load alters with age. Any culture and PCR amplification methods cannot by their nature be quantitative as they rely on different growth rates and are subject to potential PCR primer biases. Patients using hormonal medication for example progesterone only contraceptive pill or those using a progesterone coil were excluded as the effect of progesterones on the microbiome is uncertain. Progesterones may induce changes in the urogenital tract consistent with a pregnancy state which may alter the urinary microbiome. Those on HRT (2) were excluded from the study because it would be expected that the HRT might recreate the microbiome seen in premenopausal women. The exact effect of different exogenous hormones is uncertain but it seemed sensible to exclude those patients taking hormones which might alter the bladder microbiome. 
Lactobacillus has been established as a component of the bladder microbiome of women [7] although its variation with age has not previously been noted. Lactobacillus is also a key component of the normal vaginal microbiome. This study used clean catch MSU samples but there is debate as to whether MSUs or CSUs are the best way to sample the bladder microbiome [13]. Asymptomatic patients with no urinary symptoms, in common with those in other studies, found CSUs unacceptable [11]. There could be concerns that by using clean catch midstream urine samples there might be vaginal contamination and that these results may be confounded by the decline in vaginal lactobacillus with age. However, MSUs have been used extensively in the past and may continue to be used until the benefit of one technique over the other is established [2]. CSUs may be more easily justified in symptomatic patients.

Potential weaknesses of the study include the size of the study and the use of MSU samples. The study might fail to identify small differences in the bladder microbiome. Second generation sequencing in a larger sample might provide some further support to the findings of the study but there are also limitations with this methodology and analysis of the results produced by this method is complex. The effect of exogenous hormones on the bladder microbiome is uncertain and certain presumptions have been made in assessing the effect that might be supposed. Further study in this area is warranted although the methodology to achieve this is difficult. In general patients taking exogenous hormones were excluded to reduce the risk of this affecting the results.

This study characterises the bladder microbiome in health in different age groups. As expected the microbiome does alter with age. This study shows the crucial importance that age has on the bladder microbiome in healthy patients without bladder symptoms. Any 
further research in this area should take account of the natural variation that occurs with age before any variations in the bladder microbiome can be attributed to a disease process. This study will allow us to compare the bladder microbiome in health to that seen in different disease states such as OAB. Many organisms live in the bladder and exist without giving symptoms. Further work is important to identify the relevance of different organisms to health and different disease states. 


\section{References}

1. Hilt EE, McKinley K, Pearce MM, Rosenfeld AB et al. Urine is not sterile: use of enhanced urine culture techniques to detect resident bacterial flora in the adult female bladder. J Clin Microbiol 2014;52:871-6.

2. Lewis, DA, Brown R, Williams J et al. The human urinary microbiome: bacterial DNA in voided urine of asymptomatic adults. Front Cell Infect Microbiol 2013;3:41.

3. Pearce $M M$, Hilt EE, Rosenfeld $A B$, Zilliox $M J$ et al. The female urinary microbiome: $a$ comparison of women with and without urgency urinary incontinence. MBio 2014 doi: 10.1128/mBio.01283-14.

4. Brubaker L, Nager CW, Richter HE et al. Urinary bacteria in adult women with urgency urinary incontinence. Int Urogynecol J 2014;25:1179-84.

5. Kumar M, Babaei P, Ji B, Nielsen J. Human gut microbiota and healthy aging: Recent developments and future prospective. Nutrition and Healthy Aging. 2016;4(1):3-16.

6. Brotman RM, Shardell MD, Gajer P, et al. Association between the vaginal microbiota, menopause status and signs of vulvovaginal atrophy. Menopause (New York, NY). 2014;21(5):450-458.

7. Curtiss N, Balachandran A, Krska L, Peppiatt-Wildman C, Wildman S, Duckett J.A case controlled study examining the bladder microbiome in women with Overactive Bladder (OAB) and healthy controls. Eur J Obstet Gynecol Reprod Biol 2017;214:3135.

8. Machin D, Campbell M, Fayers P, Pinol A. 1997. Sample size tables for clinical studies. Blackwell. Oxford. 
9. Brubaker $\mathrm{L}$, Wolfe AJ. The female urinary microbiota, urinary health and common urinary disorders. Ann Transl Med 2017;5:34.

10. Karstens L, Asquith M, Davin S et al. Does the Urinary Microbiome Play a Role in Urgency Urinary Incontinence and its severity? Front Cell Infect Microbiol.2016; 6:78.

11. Khasriya R, Sathiananthamoorthy S, Ismail S et al. Spectrum of bacterial colonization associated with urothelial cells from patients with chronic lower urinary tract symptoms. J Clin Microbiol 2013;51:2054-62.

12. Biagi E, Candela M, Fairweather-Tait S et al. Ageing of the human metaorganism: the microbial counterpart. Age. 2012;34(1):247-267.

13. Moore $\mathrm{KH}$, Malykhina AP What is the role of covert infection in detrusor overactivity, and other LUTD? ICI-RS 2013. Neurourol Urodyn 2014;33:606-10. 
Figure 1. Plot of age against total number of genera identified

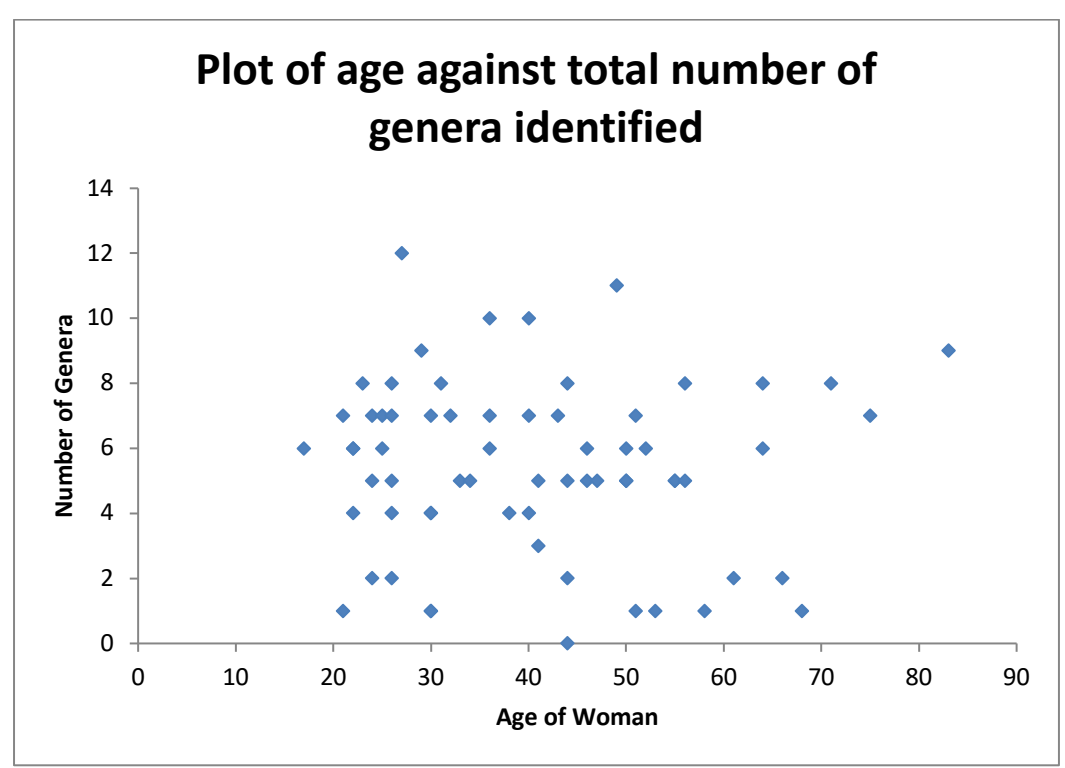




\section{Figure 2}

Diagram depicting the distribution of genera by menopausal status (species present)

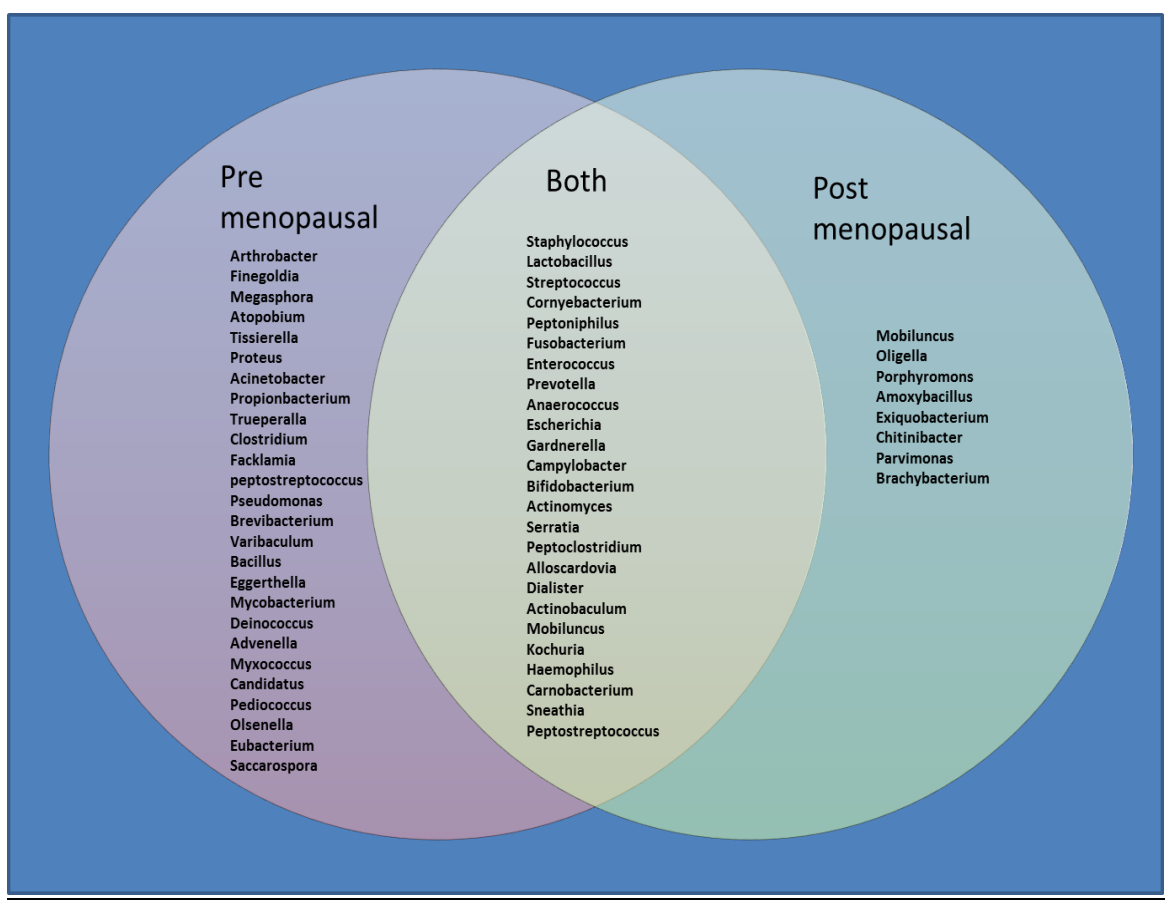


Table 1: Results depicted by age range

\begin{tabular}{|l|l|ll|lc|l|l|}
\hline Age & Number & \multicolumn{2}{|l|}{$\begin{array}{l}\text { Lactobacillus } \\
(\%)\end{array}$} & $\begin{array}{l}\text { total number of } \\
\text { genera (range) }\end{array}$ & \multicolumn{2}{l}{$\begin{array}{l}\text { Menopausal } \\
(\%)\end{array}$} \\
\hline $10-19$ & 1 & 1 & $(100 \%)$ & 6.0 & & $0 \quad(0 \%)$ \\
\hline $20-29$ & 23 & 16 & $(69.9 \%)$ & 5.7 & $(1-12)$ & $0 \quad(0 \%)$ \\
\hline $30-39$ & 17 & 11 & $(64.7 \%)$ & 5.4 & $(1-10)$ & $0 \quad(0 \%)$ \\
\hline $40-49$ & 16 & 10 & $(62.5 \%)$ & 5.4 & $(0-11)$ & $0 \quad(0 \%)$ \\
\hline $50-59$ & 14 & 5 & $(35.7 \%)$ & 4.8 & $(1-8)$ & $11(78.6 \%)$ \\
\hline $60-69$ & 5 & 1 & $(20 \%)$ & 3.8 & $(1-8)$ & $5 \quad(100)$ \\
\hline $70-79$ & 2 & 0 & $(0 \%)$ & 7.5 & $(1-2)$ & $2 \quad(100)$ \\
\hline $80-89$ & 1 & 0 & $(0 \%)$ & 9.0 & & $1(100)$ \\
\hline
\end{tabular}

